\title{
Qualidade de vida de mestrandos em Odontologia
}

\author{
Maria Imaculada de Queiroz Rodrigues*; Raul Anderson Domingues Alves da Silva**; Myrna Maria \\ Arcanjo Frota Barros***; Paulo Goberlanio de Barros Silva***; Cinthia Nara Gadelha Teixeira****; \\ Maria Eneide Leitão de Almeida*****
}

* Graduanda, Curso de Odontologia, Universidade Federal do Ceará

** Mestrando em Clínica Odontológica, Universidade Federal do Ceará

*** Doutor(a) em Clínica Odontológica, Universidade Federal do Ceará

**** Doutoranda em Odontologia, Universidade Federal do Maranhão

***** Professora, Programa de Pós-graduação em Odontologia, Universidade Federal do Ceará

Recebido em 15/07/2019. Aprovado em 21/04/2020.

\begin{abstract}
RESUMO
O objetivo deste estudo foi avaliar a Qualidade de Vida (QV) dos mestrandos em Odontologia da Universidade Federal do Ceará. O estudo foi transversal, do tipo quantitativo e a coleta de dados ocorreu no período de agosto de 2015 a maio de 2016. Foram investigadas variáveis sociodemográficas e a QV foi mensurada utilizando o instrumento Whoqol-bref. Foi realizada análise bivariada e regressão logística multinominal. Participaram do estudo 50 discentes, sendo 29 do sexo feminino (58\%); 37 eram solteiros (74\%) e 47 não possuíam filhos $(94 \%)$. Os resultados mostraram que na análise de QV 60\% da amostra apresentou satisfação. Já quanto aos domínios, 82\% obteve insatisfação/indiferença no domínio Físico, enquanto $76 \%$ mostraram-se satisfeitos quanto ao domínio Relações Sociais. Significância estatística foi encontrada quando associada a participação em grupo de atividades sociais ao domínio Meio Ambiente $(\mathrm{p}=0,016)$. A maior parte da amostra mostrou-se satisfeita quanto à qualidade de vida, enquanto o domínio Físico apresentou maior percentual de insatisfação/indiferença e o de Relações Sociais o maior percentual de satisfação.

Descritores: Educação de Pós-Graduação. Odontologia. Religião. Qualidade de Vida.
\end{abstract}




\section{INTRODUÇÃO}

A Qualidade de Vida (QV) é definida pela Organização Mundial da Saúde (OMS) como a percepção do indivíduo de sua posição na vida, no contexto da cultura e sistemas de valores nos quais vive e em relação aos seus objetivos, expectativas, padrões e preocupações ${ }^{1}$.

A pós-graduação no Brasil tem como forte característica um elevado nível de exigência de seus pós-graduandos durante todo o processo de formação ${ }^{2}$. O curso de mestrado em si já se configura como um evento macro estressor e durante o período de curso o aluno é, ainda, exposto a diversas outras situações que podem afetar sobremaneira a sua $\mathrm{QV}$, principalmente de forma negativa ${ }^{3}$.

Em sua rotina, os pós-graduandos passam a exercer diversas atividades para cumprir a gama de exigências de seus programas de pósgraduação e orientadores, como cursar disciplinas; ministrar aulas; participar de grupos de pesquisa e de estudo, colaborar nas pesquisas de outros pós-graduandos, orientar estudantes de graduação, participar de eventos científicos e divulgar resultados de pesquisa por meio de apresentações, artigos e capítulos de livros; além de desenvolverem suas pesquisas e elaborar sua dissertação/tese, entre outras. Assim, diante das numerosas exigências e tarefas a serem cumpridas, em conjunto a vida pessoal, uma sobrecarga pode acabar por influenciar de forma negativa a sua qualidade de vida ${ }^{4}$.

Um dos instrumentos criados para avaliar qualidade de vida de um indivíduo foi o WHOQOL-100, desenvolvido pela Divisão de Saúde Mental da Organização Mundial da Saúde (OMS). Trata-se de um questionário com 100 questões, validado em diversos países, com diversas publicações no meio científico ${ }^{1}$. Para tornar a sua aplicação mais rápida, foi desenvolvido o WHOQOL-bref10, que é composto por 26 questões, sendo duas sobre a autoavaliação da QV e 24 representando cada uma das facetas do WHOQOL-100. Pesquisas utilizando o WHOQOL-bref e outros parâmetros para medir a qualidade de vida já são realizadas há mais de 30 anos $^{5}$.

São escassas as pesquisas que avaliam qualidade de vida de pós-graduandos em Odontologia, permitindo a identificação de fatores sociodemográficos que possam se relacionar com o seu desempenho e sua qualidade de vida. A avaliação de seus níveis de satisfação e fatores associados são de suma importância para o reconhecimento da atual realidade de vida dessa população, assim como na criação de planos de ação capazes de transformá-la, criando um ambiente favorável ao aprendizado e à formação acadêmica. Dessa forma, o objetivo da presente pesquisa é avaliar a Qualidade de Vida (QV) dos alunos de mestrado em Odontologia do Programa de Pós-graduação em Odontologia da Universidade Federal do Ceará (PPGO-UFC).

\section{METODOLOGIA}

A pesquisa é caracterizada como estudo transversal do tipo quantitativo. A coleta de dados ocorreu no período de agosto de 2015 a maio de 2016, sendo os dados coletados por três pesquisadoras. A calibração não foi necessária devido ao alto nível intelectual dos pesquisados e à fácil aplicabilidade do instrumento. Participaram do estudo 50 mestrandos regularmente matriculados no programa, compondo $100 \%$ do universo pesquisado.

O questionário aplicado foi composto por duas partes, a primeira continha dados sociodemográficos gerais dos discentes, como sexo, estado civil, se possuía filhos, além das questões que envolviam religiosidade, e participação em grupo de atividades sociais. Já a segunda, era composta pelo questionário abreviado sobre QV da Organização Mundial da 
Saúde, o WHOQOL-bref ${ }^{6}$, que contém 26 questões, sendo as duas primeiras sobre a autoavaliação da QV do indivíduo e as demais divididas em quatro domínios: Físico, Psicológico, Relações Sociais e Meio Ambiente ${ }^{6}$.

As questões distribuídas por domínios são:

Físico (sete questões sobre dor e desconforto, energia e fadiga, sono e repouso, mobilidade, atividades da vida cotidiana, uso de medicamentos e capacidade para o trabalho); Psicológico (seis questões sobre sentimentos positivos e negativos, pensar e aprender, memória e concentração, imagem corporal e espiritualidade); Relações Sociais (três questões sobre relações pessoais, suporte social e atividade sexual); Meio Ambiente (oito questões sobre segurança física e proteção, ambiente do lar, recursos financeiros, disponibilidade $\mathrm{e}$ qualidade de cuidados de saúde e sociais, oportunidades de adquirir novas informações e habilidades, atividades de lazer, ambiente físico e transporte) ${ }^{6}$.

Todas as questões apresentam resposta em escala do tipo Likert, tendo cinco opções para cada uma delas. Para o preenchimento do questionário devem ser considerados os 15 dias anteriores ${ }^{6}$.

Os participantes responderam ao questionário após a leitura e assinatura do Termo de Consentimento Livre e Esclarecido. O estudo foi aprovado pelo Comitê de Ética e Pesquisa da UFC (CAAE 45227015.7.0000.5054).

Os dados coletados foram digitados no Microsoft Excel (Redmond, WA, EUA) e posteriormente analisados no software Statistical Package for the Social Sciences (San Diego, CA, EUA), considerando intervalo de confiança de $95 \%$ e significância com $\mathrm{p}<0,05$.

Como recomendação da sintaxe do próprio WHOQOL group, os escores obtidos foram transformados em uma escala linear que variou de 0-100, sendo estes, respectivamente, os valores menos e mais favoráveis de $\mathrm{QV}^{6}$. Para melhor interpretação dos dados de QV, utilizouse uma escala adaptada, sendo assim categorizada: valores entre 0 e 40 estavam no nível de insatisfação; de 41 a 69 , estavam no nível de indefinição; e, a partir de 70, estavam no nível de satisfação ${ }^{7}$.

Foram utilizados testes paramétricos (teste t de Student) e não paramétricos (chi quadrado de Pearson), segundo as características das variáveis do estudo. Análise bivariada foi realizada para as associações entre as variáveis, sendo calculadas, também, o modelo de regressão logística multinominal. $\mathrm{Na}$ análise, as variáveis de QV e dos domínios foram agrupadas em insatisfeito/indiferente e satisfeito.

\section{RESULTADOS}

Participaram desse estudo 50 mestrandos, sendo $58 \%$ do sexo feminino; $74 \%$ eram solteiros e $94 \%$ afirmaram não ter filhos. Quanto à prática religiosa, $84 \%$ dos alunos praticavam alguma religião, 66\% consideravam-se religiosos; $80 \%$ afirmaram ser a religião o sentido da vida e $70 \%$ não participavam de atividade social (tabela 1).

A qualidade de vida foi mensurada no intervalo de insatisfação/indiferença por $60 \%$ $(\mathrm{n}=30)$ dos entrevistados, sendo o domínio Relações Sociais o de maior percentual (76\%, 38 entrevistados) no intervalo de satisfação, enquanto o domínio Físico apresentou o maior percentual de insatisfação/indiferença $(82 \%, 41$ entrevistados). Já os domínios Psicológico e Meio Ambiente obtiveram, respectivamente, $56 \%(n=28)$ e $62 \%(n=31)$ de percentual de insatisfação/indiferença pelos entrevistados.

Não houve diferença estatística entre as variáveis de religiosidade e os domínios Físico (tabela 2), Psicológico (tabela 3) e Relações Sociais (tabela 4).

A tabela 5 traz a associação entre as variáveis sociodemográficas e o domínio Meio 
Ambiente. Significância estatística foi Ambiente e a variável participação em grupo de encontrada ao associar o domínio Meio atividade social $(\mathrm{p}=0,016)$.

Tabela 1. Dados sociodemográficos dos mestrandos

\begin{tabular}{|c|c|c|}
\hline Variáveis & $\mathbf{n}$ & $\%$ \\
\hline \multicolumn{3}{|l|}{ Sexo } \\
\hline Masculino & 21 & 42,0 \\
\hline Feminino & 29 & 58,0 \\
\hline \multicolumn{3}{|l|}{ Estado civil } \\
\hline Solteiro & 37 & 74,0 \\
\hline Casado & 13 & 26,0 \\
\hline \multicolumn{3}{|l|}{ Filhos } \\
\hline Sim & 3 & 6,0 \\
\hline Não & 47 & 94,0 \\
\hline \multicolumn{3}{|c|}{ Prática de alguma religião } \\
\hline Sim & 42 & 84,0 \\
\hline Não & 8 & 16,0 \\
\hline \multicolumn{3}{|c|}{ Frequência da prática religiosa } \\
\hline Nunca & 6 & 12,0 \\
\hline Raramente & 7 & 14,0 \\
\hline Às vezes & 19 & 38,0 \\
\hline Sempre & 18 & 36,0 \\
\hline \multicolumn{3}{|c|}{ Considera-se religioso } \\
\hline Sim & 33 & 66,0 \\
\hline Não & 17 & 34,0 \\
\hline \multicolumn{3}{|c|}{ Religião como sentido da vida } \\
\hline Sim & 40 & 80,0 \\
\hline Não & 10 & 20,0 \\
\hline \multicolumn{3}{|c|}{ Participação em grupo de atividade social } \\
\hline Sim & 15 & 30,0 \\
\hline Não & 35 & 70,0 \\
\hline
\end{tabular}


Tabela 2. Associação entre o domínio Físico e os dados sociodemográficos dos mestrandos

\begin{tabular}{|c|c|c|c|c|}
\hline \multirow[b]{2}{*}{ Variáveis } & \multicolumn{2}{|l|}{ Domínio Físico } & \multirow[b]{2}{*}{ p-Valor ${ }^{\mathbf{a}}$} & \multirow[b]{2}{*}{ p-Valor ${ }^{b}$} \\
\hline & Insatisfeito/Indiferente & Satisfeito & & \\
\hline \multicolumn{5}{|l|}{ Sexo } \\
\hline Masculino & $18(43,9 \%)$ & $3(33,3 \%)$ & 0,716 & 0,990 \\
\hline Feminino & $23(56,1 \%)$ & $6(66,7 \%)$ & & \\
\hline \multicolumn{5}{|l|}{ Estado civil } \\
\hline Solteiro & $32(78,0 \%)$ & $5(55,6 \%)$ & 0,164 & 0,975 \\
\hline Casado & $9(22,0 \%)$ & $4(44,4 \%)$ & & \\
\hline \multicolumn{5}{|l|}{ Filhos } \\
\hline Sim & $2(4,9 \%)$ & $1(11,1 \%)$ & 0,476 & 0,997 \\
\hline Não & $39(95,1 \%)$ & $8(88,9 \%)$ & & \\
\hline \multicolumn{5}{|c|}{ Prática de alguma religião } \\
\hline Sim & $37 *(90,2 \%)$ & $5(55,6 \%)$ & 0,026 & 0,998 \\
\hline Não & $4(9,8 \%)$ & $4 *(44,4 \%)$ & & \\
\hline \multicolumn{5}{|c|}{ Frequência da prática religiosa } \\
\hline Nunca & $3(7,3 \%)$ & $3 *(33,3 \%)$ & 0,033 & 0,961 \\
\hline Raramente & $5(12,2 \%)$ & $2(22,2 \%)$ & & \\
\hline Às vezes & $15(36,6 \%)$ & $4(44,4 \%)$ & & \\
\hline Sempre & $18 *(43,9 \%)$ & $0(0,0 \%)$ & & \\
\hline \multicolumn{5}{|c|}{ Considera-se religioso } \\
\hline Sim & $29(70,7 \%)$ & $4(44,4 \%)$ & 0,242 & 1,000 \\
\hline Não & $12(29,3 \%)$ & $5(55,6 \%)$ & & \\
\hline \multicolumn{5}{|c|}{ Religião como sentido da vida } \\
\hline Sim & $34(82,9 \%)$ & $6(66,7 \%)$ & 0,358 & 0,975 \\
\hline Não & $7(17,1 \%)$ & $39(33,3 \%)$ & & \\
\hline \multicolumn{5}{|c|}{ Participação em grupo de atividade social } \\
\hline Sim & $32(78,0 \%)$ & $5(55,6 \%)$ & 0,247 & 0,996 \\
\hline Não & $9(22,0 \%)$ & $4(44,4 \%)$ & & \\
\hline
\end{tabular}

aTeste Exato de Fisher ou Qui-quadrado de Pearson; *p<0,05;

${ }^{\mathrm{b}}$ Regressão Logística Multinomial; $\uparrow \mathrm{p}<0,05$; 
Tabela 3. Associação entre o domínio Psicológico e os dados sociodemográficos dos mestrandos

\begin{tabular}{|c|c|c|c|c|}
\hline \multirow[b]{2}{*}{ Variáveis } & \multicolumn{2}{|c|}{ Domínio Psicológico } & \multirow[b]{2}{*}{ p-Valor ${ }^{\mathbf{a}}$} & \multirow[b]{2}{*}{ p-Valor ${ }^{b}$} \\
\hline & Insatisfeito/Indiferente & Satisfeito & & \\
\hline \multicolumn{5}{|l|}{ Sexo } \\
\hline Masculino & $9(32,1 \%)$ & $12 \dagger(54,5 \%)$ & 0,111 & 0,017 \\
\hline Feminino & $19 \dagger(67,9 \%)$ & $10(45,5 \%)$ & & \\
\hline \multicolumn{5}{|l|}{ Estado civil } \\
\hline Solteiro & $22(78,6 \%)$ & $15(68,2 \%)$ & 0,406 & 0,195 \\
\hline Casado & $6(21,4 \%)$ & $7(31,8 \%)$ & & \\
\hline \multicolumn{5}{|l|}{ Filhos } \\
\hline Sim & $1(3,6 \%)$ & $2(9,1 \%)$ & 0,576 & 0,995 \\
\hline Não & $27(96,4 \%)$ & $20(90,9 \%)$ & & \\
\hline \multicolumn{5}{|c|}{ Prática de alguma religião } \\
\hline Sim & $24(85,7 \%)$ & $18(81,8 \%)$ & 0,718 & 0,997 \\
\hline Não & $4(14,3 \%)$ & $4(18,2 \%)$ & & \\
\hline \multicolumn{5}{|c|}{ Frequência da prática religiosa } \\
\hline Nunca & $4(14,3 \%)$ & $2(9,1 \%)$ & 0,452 & 0,996 \\
\hline Raramente & $2(7,1 \%)$ & $5(22,7 \%)$ & & \\
\hline Às vezes & $11(39,3 \%)$ & $8(36,4 \%)$ & & \\
\hline Sempre & $11(39,3 \%)$ & $7(31,8 \%)$ & & \\
\hline \multicolumn{5}{|c|}{ Considera-se religioso } \\
\hline Sim & $21(75,0 \%)$ & $12(54,5 \%)$ & 0,130 & 0,309 \\
\hline Não & $7(25,0 \%)$ & $10(45,5 \%)$ & & \\
\hline \multicolumn{5}{|c|}{ Religião como sentido da vida } \\
\hline Sim & $24(85,7 \%)$ & $16(72,7 \%)$ & 0,254 & 0,585 \\
\hline Não & $4(14,3 \%)$ & $6(27,3 \%)$ & & \\
\hline \multicolumn{5}{|c|}{ Participação em grupo de atividade social } \\
\hline $\operatorname{Sim}$ & $10(35,7 \%)$ & $5(22,7 \%)$ & 0,320 & 0,222 \\
\hline Não & $18(64,3 \%)$ & $17(77,3 \%)$ & & \\
\hline
\end{tabular}

${ }^{\mathrm{a}}$ Teste Exato de Fisher ou Qui-quadrado de Pearson; ${ }^{*} \mathrm{p}<0,05$;

${ }^{\mathrm{b}}$ Regressão Logística Multinomial; $\uparrow \mathrm{p}<0,05$; 
Tabela 4. Associação entre o domínio Relações Sociais e os dados sociodemográficos dos mestrandos

\begin{tabular}{|c|c|c|c|c|}
\hline \multirow[b]{2}{*}{ Variáveis } & \multicolumn{2}{|c|}{ Domínio Relações Sociais } & \multirow[b]{2}{*}{ p-Valor ${ }^{a}$} & \multirow[b]{2}{*}{ p-Valor } \\
\hline & Insatisfeito/Indiferente & Satisfeito & & \\
\hline \multicolumn{5}{|l|}{ Sexo } \\
\hline Masculino & $6(50,0 \%)$ & $15(39,5 \%)$ & 0,520 & 0,297 \\
\hline Feminino & $6(50,0 \%)$ & $23(60,5 \%)$ & & \\
\hline \multicolumn{5}{|l|}{ Estado civil } \\
\hline Solteiro & $11(91,7 \%)$ & $26(68,4 \%)$ & 0,147 & 0,988 \\
\hline Casado & $1(8,3 \%)$ & $12(31,6 \%)$ & & \\
\hline \multicolumn{5}{|l|}{ Filhos } \\
\hline Sim & $1(8,3 \%)$ & $2(5,3 \%)$ & 1,000 & 0,987 \\
\hline Não & $11(91,7 \%)$ & $36(94,7 \%)$ & & \\
\hline \multicolumn{5}{|c|}{ Prática de alguma religião } \\
\hline Sim & $10(83,3 \%)$ & $32(84,2 \%)$ & 1,000 & 0,988 \\
\hline Não & $2(16,7 \%)$ & $6(15,8 \%)$ & & \\
\hline \multicolumn{5}{|c|}{ Frequência da prática religiosa } \\
\hline Nunca & $1(8,3 \%)$ & $5(13,2 \%)$ & 0,286 & 0,110 \\
\hline Raramente & $3(25,0 \%)$ & $4(10,5 \%)$ & & \\
\hline Às vezes & $6(50,0 \%)$ & $13(34,2 \%)$ & & \\
\hline Sempre & $2(16,7 \%)$ & $16(42,1 \%)$ & & \\
\hline \multicolumn{5}{|c|}{ Considera-se religioso } \\
\hline Sim & $7(58,3 \%)$ & $26(68,4 \%)$ & 0,728 & 0,190 \\
\hline Não & $5(41,7 \%)$ & $12(31,6 \%)$ & & \\
\hline \multicolumn{5}{|c|}{ Religião como sentido da vida } \\
\hline Sim & $8(66,7 \%)$ & $32(84,2 \%)$ & 0,185 & 0,984 \\
\hline Não & $4(33,3 \%)$ & $6(15,8 \%)$ & & \\
\hline \multicolumn{5}{|c|}{ Participação em grupo de atividade social } \\
\hline Sim & $5(41,7 \%)$ & $10(26,3 \%)$ & 0,312 & 0,068 \\
\hline Não & $7(58,3 \%)$ & $28(73,7 \%)$ & & \\
\hline
\end{tabular}

Teste Exato de Fisher ou Qui-quadrado de Pearson; *p<0,05;

${ }^{\mathrm{b}}$ Regressão Logística Multinomial; $\uparrow \mathrm{p}<0,05$; 
Tabela 5. Associação entre o domínio Meio Ambiente e os dados sociodemográficos dos mestrandos

\begin{tabular}{|c|c|c|c|c|}
\hline \multirow[b]{2}{*}{ Variáveis } & \multicolumn{2}{|c|}{ Domínio Meio Ambiente } & \multirow[b]{2}{*}{ p-Valor ${ }^{a}$} & \multirow[b]{2}{*}{ p-Valor ${ }^{b}$} \\
\hline & Insatisfeito/Indiferente & Satisfeito & & \\
\hline \multicolumn{5}{|l|}{ Sexo } \\
\hline Masculino & $13(41,9 \%)$ & $8(42,1 \%)$ & 1,000 & 0,152 \\
\hline Feminino & $18(58,1 \%)$ & $11(57,9 \%)$ & & \\
\hline \multicolumn{5}{|l|}{ Estado civil } \\
\hline Solteiro & $25(80,6 \%)$ & $12(63,2 \%)$ & 0,199 & 0,945 \\
\hline Casado & $6(19,4 \%)$ & $7(36,8 \%)$ & & \\
\hline \multicolumn{5}{|l|}{ Filhos } \\
\hline Sim & $1(3,2 \%)$ & $29(10,5 \%)$ & 0,549 & 0,382 \\
\hline Não & $30(96,8 \%)$ & $17(89,5 \%)$ & & \\
\hline \multicolumn{5}{|c|}{ Prática de alguma religião } \\
\hline Sim & $29 *(93,5 \%)$ & $13(68,4 \%)$ & 0,041 & 0,996 \\
\hline Não & $2(6,5 \%)$ & $6^{*}(31,6 \%)$ & & \\
\hline \multicolumn{5}{|c|}{ Frequência da prática religiosa } \\
\hline Nunca & $2(6,5 \%)$ & $4(21,1 \%)$ & 0,447 & 0,997 \\
\hline Raramente & $4(12,9 \%)$ & $3(15,8 \%)$ & & \\
\hline Às vezes & $13(41,9 \%)$ & $6(31,6 \%)$ & & \\
\hline Sempre & $12(38,7 \%)$ & $6(31,6 \%)$ & & \\
\hline \multicolumn{5}{|c|}{ Considera-se religioso } \\
\hline Sim & $21(67,7 \%)$ & $12(63,2 \%)$ & 0,740 & 0,977 \\
\hline Não & $10(32,3 \%)$ & $7(36,8 \%)$ & & \\
\hline \multicolumn{5}{|c|}{ Religião como sentido da vida } \\
\hline Sim & $27(87,1 \%)$ & $13(68,4 \%)$ & 0,150 & 0,825 \\
\hline Não & $4(12,9 \%)$ & $6(31,6 \%)$ & & \\
\hline \multicolumn{5}{|c|}{ Participação em grupo de atividade social } \\
\hline Sim & $12 \dagger(38,7 \%)$ & $3(15,8 \%)$ & 0,086 & 0,016 \\
\hline Não & $19(61,3 \%)$ & $16 \dagger(84,2 \%)$ & & \\
\hline
\end{tabular}

aTeste Exato de Fisher ou Qui-quadrado de Pearson; ${ }^{*} \mathrm{p}<0,05$;

${ }^{\mathrm{b}}$ Regressão Logística Multinomial; $\uparrow \mathrm{p}<0,05$; 


\section{DISCUSSÃO}

A maioria dos participantes foi classificada no intervalo de insatisfação/indiferença quanto à $\mathrm{QV}$, diferindo de um estudo realizado com estudantes de graduação em Odontologia no Ceará ${ }^{7}$ e de outro realizado com uma população adulta do Distrito Federal ${ }^{8}$. Tal resultado pode ser justificado por serem mestrandos submetidos a altas exigências na pós-graduação, como pesquisar, ministrar aulas, necessidade de publicação, preocupação com as condições financeiras, além de ter de lidar com a frequente ambiguidade de expectativas do orientador ${ }^{9}$.

O domínio Relações Sociais foi o que obteve maior percentual de satisfação quanto à qualidade de vida. Esse resultado pode ser explicado por envolver apenas três aspectos, relações pessoais, suporte social e atividade sexual, tendo o mestrando a necessidade de um suporte social consistente para a realização do curso. Além disso, uma pesquisa realizada com pós-graduandos em Enfermagem encontrou uma relação positiva entre o relacionamento interpessoal com os membros do corpo docente do curso, que gerou um impacto positivo na qualidade de vida de sua amostra ${ }^{10}$. Essa relação satisfatória, muitas vezes, é tida como favorecedora de qualidade de vida para esses estudantes, além de servir como oportunidade de desenvolver novas habilidades e aprendizados, assim como servirem de suporte intelectual e emocional no enfrentamento das dificuldades ao longo do curso ${ }^{11-12}$.

O domínio Físico teve maior percentual de insatisfação na presente pesquisa, o que pode ser explicado pelo próprio esforço de se estar cursando uma pós-graduação, que na maioria das vezes exige alta produção científica e prazos para conclusão do curso. Isso tem causado uma dificuldade de conciliar a vida acadêmica com a vida pessoal, exigindo esforço físico e mental cada vez maior do pós-graduando ${ }^{13-14}$. Além disso, uma pesquisa desenvolvida com estudantes de graduação e pós-graduação em Odontologia também encontrou resultados semelhantes, onde o domínio físico apresentou menor média de satisfação do que os outros domínios. Segundo os autores, dentre as questões que correspondem a esse domínio, a questão com maior destaque negativo foi a que se referia a posse de energia para executar tarefas do seu diaa-dia, o que pode estar relacionada com as exigências e alta carga horária dos cursos ${ }^{15}$.

Não foi encontrada associação entre as variáveis de religiosidade e os domínios Físico, Psicológico e Relações Sociais. Ao todo, esses três domínios envolvem dezesseis aspectos, que vão desde o sono e o repouso, passando pelos sentimentos positivos e negativos da vida, até a atividade sexual, o que é condizente com os resultados de estudo que constatou que a religiosidade é mais procurada pelas pessoas em casos de doenças graves ${ }^{16}$. Outro estudo retratou que as dimensões de espiritualidade e religiosidade estão associadas à melhor $\mathrm{QV}$, com melhores resultados para as pessoas que estão se recuperando de doença física e mental ${ }^{17},{ }^{18}$.

A participação em grupo de atividade social indicou maior insatisfação/indiferença quanto à QV, com associação significativa ao domínio Meio Ambiente. Tal resultado difere do encontrado em um estudo no qual a frequência da prática religiosa, a participação em eventos sociais e de alguma instituição religiosa esteve ligado à maior estabilidade na $\mathrm{QV}^{19}$. Esse resultado pode ser justificado por ser o mestrado um curso intenso e de curta duração, com a divisão do tempo entre as atividades acadêmicas, profissionais e sociais gerando dificuldades na administração adequada do tempo.

Ainda assim, a literatura tem mostrado baixa satisfação na QV de estudantes de pósgraduação em diferentes cursos e realidades no Brasil e no mundo ${ }^{7,8,10,20-23}$. Além disso, os pós- 
graduandos têm mostrado maior insatisfação quanto à qualidade de vida quando comparados aos graduandos, o que sugere a interferência de características específicas desse nível de escolaridade $^{10}$. Acredita-se que a aceitação desse estilo de vida e os impactos na sua qualidade de vida esteja na crença de que tudo não passa de uma etapa de transição para uma possível melhora, e que a continuidade nesse tipo de vivência também tem seu lado positivo: o privilégio de cursar mais um nível de ensino, que leva ao reconhecimento pessoal e profissional, aprimoramento e crescimento profissional e à chance de melhores oportunidades financeiras ${ }^{24}$.

A grande disputa no mercado de trabalho, a constante busca por melhor qualificação profissional e o investimento em programas de pós-graduação acabaram por transformar a atual realidade, estimulado a alta demanda de produção científica que resulta em maiores investimentos das agências de fomento, sem levar em conta os consequentes impactos na QV.

Algumas limitações estão presentes nesse estudo: o seu delineamento transversal, que não permite inferências de causalidade; sua amostra restrita a uma população localizada que limita a generalização dos seus achados; além do não estabelecimento de uma precedência temporal sobre os desfechos avaliados. Investigações futuras que envolvam outros aspectos relacionando estudantes de mestrado, religiosidade e qualidade de vida, como estudos longitudinais e a investigação de outros aspectos que envolvam a religiosidade são necessários.

\section{CONCLUSÃO}

A maior parte da amostra apresentou satisfação geral quanto à QV. Entre os domínios, o Físico apresentou maior percentual de insatisfação/indiferença, enquanto o de Relações Sociais apresentou maior percentual de satisfação. Quanto às características sociodemográficas, somente a variável "prática em grupo de atividade social" influenciou na QV dos mestrandos, nos aspectos que norteiam o domínio Meio Ambiente.

\section{ABSTRACT}

The objective of this study was to evaluate the Quality of Life (QOL) of master's degree students in Dentistry of the Federal University of Ceará. This was a cross-sectional, quantitativetype study and data collection occurred from August 2015 to May 2016. Sociodemographic variables were investigated and QOL was measured using the Whoqol-bref instrument. Bivariate analysis and multinomial logistic regression were performed. Fifty students participated in the study, 29 were female (58\%); 37 were single $(74 \%)$ and 47 had no children (94\%). The results showed that in the QOL analysis, $60 \%$ of the sample was satisfied. As for the domains, $82 \%$ obtained dissatisfaction/ indifference in the Physical domain, while $76 \%$ were satisfied with the Social Relations domain. Statistical significance was found when associated with participation in a group of social activities in the Environment domain ( $p=0,016)$. The vast majority of the sample was satisfied with the quality of life, while the Physical domain had the highest percentage of dissatisfaction/indifference and the Social Relationship the highest percentage of satisfaction.

DESCRIPTORS: Education, Graduate. Dentistry. Religion. Quality of Life.

\section{REFERÊNCIAS}

1. World Health Organization. Division of Mental Health and Prevention of Substance Abuse. WHOQOL: measuring quality of life. Geneva. World Health Organization. 1997

2. Zanella AV. Atividade criadora, produção de conhecimentos e formação de pesquisadores: algumas reflexões. Psicol Soc. 2004 ; 16(1): 135-45. 
3. Santos AF, Júnior AA. Estresse e estratégias de enfrentamento em mestrandos de ciências da saúde. Psicol Reflex Crit. 2007; 20(1): 104-13.

4. Faro A. Um modelo explicativo para o bemestar subjetivo: estudo com mestrandos e doutorandos no Brasil. Psicol Reflex Crit. 2013; 26(4):654-62

5. Fleck MPA, Louzada S, Xavier M, Chachamovich E, Vieira G, Santos L et al. Aplicação da versão em português do instrumento abreviado de avaliação da qualidade de vida "WHOQOL-Bref". Rev Saúde Pública. 2000; 34(2):178-83.

6. Whoqol. The Whoqol Group. Sintaxe SPSS - WHOQOL - bref Questionnaire. [acesso em 17 Dez 2015]. Disponível em: http://www.ufrgs.br/psiq/whoqol86.html.

7. Brito DP, Oliveira LMR, Braga SR, Nuto SAS, Viana FAC. Avaliação da qualidade de vida de acadêmicos de Odontologia do estado do Ceará. Coleção Pesquisa em Educação Física. 2012; 11(3):41-50.

8. Gomes JRAA, Hamann EM, Gutierrez MMU. Aplicação do WHOQOL-BREF em segmento da comunidade como subsídio para ações de promoção da saúde. Rev. bras. epidemiol. 2014; 17(2):495-516.

9. Hyun JK, Quinn BC, Madon T, Lustig S. Graduate student mental health: needs assessment and utilization of counseling services. J Coll Student Dev. 2006; 47(3):247-66.

10. Galdino MJQ, Martins JT, Ribeiro RP, Scholze AR, Tsukamoto SAS, Haddad MCFL. Quality of life of masters and doctoral nursing students. Rev. Eletr. Enf. 2018 ;20:v20a41.

11. Nehls N, Barber G, Rice E. Pathways to the PhD in Nursing: An Analysis of Similarities and Differences. J Prof Nurs. 2016; 32(3):163-72.
12. Halse C, Bansel P. The learning alliance: ethics in doctoral supervision. Oxford Rev Educ. 2012;38(4):377-92.

13. Faro A. Estresse e estressores na pósgraduação: estudo com mestrandos e doutorandos no Brasil. Psic Teor Pesq. 2013;29(1):51-60.

14. Mendes VR, Iora JA. A opinião dos estudantes sobre as exigências da produção na pós-graduação. Rev Bras Ciênc Esporte. $2014 ; 36(1): 171-87$.

15. Amadeu, JR, Justi, MM. Qualidade de vida de estudantes de graduação e pós-graduação em Odontologia. Arch Health Invest. 2017; 6(11):540-44.

16. Miranda SL, Lanna MAL, Felippe WC. Espiritualidade, depressão e qualidade de vida no enfrentamento do câncer: estudo exploratório. Psicol Cienc Prof. 2015; 35(3):870-85.

17. Murakami R, Campos CJG. Religião e saúde mental: desafio de integrar a religiosidade ao cuidado com o paciente. Rev Bras Enferm. 2012; 65(2):361-67.

18. Fleck MPA, Borges ZN, Bolognesi G, Rocha NS. Desenvolvimento do WHOQOL, módulo espiritualidade, religiosidade e crenças pessoais. Rev Saúde Pública. 2003; 37(4):446-55.

19. Bahr HM, Harvey CD. Widowhood and perceptions of change in quality of life: evidence from the Sunshine Mine Widows. Journal of Comparative Family Studies. 1979; 10(3):411-28.

20. Vakili Z, Mohamad AR, Vakili MA. A quantitative study of quality of life (QOL) on postgraduate students in Universiti Sains Malaysia. Caspian J Appl Sci Res. 2012; 1(7), 28-32.

21. Bullappa A, Kengnal P. Assessment of quality of life of postgraduate students in a private medical college of Karnataka using 
World Health Organization quality of lifeBREF questionnaire. Int J Med Sci Public Health. 2017; 6(5):834-7.

22. Ghazanfar H, Iqbal S, Naseem S. Quality of life of post-graduate medical students working in private and public hospitals in Punjab as measured by WHOQOL-BREF questionnaire. J Pak Med Assoc. 2018; 68(6), 908-13.

23. Mendes-Rodrigues C, Ranal MA, Carvalho DVP. Postgraduate Students: an alert about Quality of Life. World J Educ. 2019; (9)1:136-44.
24. Galdino MJQ, Martins JT, Haddad MCFL, Ribeiro RP. Mestrado em enfermagem: sentimentos vivenciados por estudantes. Rev Enferm. 2016;10(2):501-7.

\section{Correspondência para:}

Raul Anderson Domingues Alves da Silva e-mail: raulanderson_alves@ hotmail.com Rua Jóckey Clube, 48-altos. Bairro Jóckey Clube 60510-115Fortaleza/CE 